\title{
Research on Digital Human Model Used in Human Factor Simulation and Evaluation of Load Carriage Equipment
}

\author{
Dayong Dong ${ }^{1}$, Lijing Wang ${ }^{2}$, Xiugan Yuan ${ }^{2}$, and Shan $\mathrm{Fu}^{1}$ \\ ${ }^{1}$ School of Aeronautics and Astronautics, \\ Shanghai Jiao Tong University, 800 Dongchuan Rd., \\ Shanghai 200240, P.R. China \\ ${ }^{2}$ School of Aeronautical Science and Engineering, \\ Beihang University, 37 Xueyuan Rd., \\ Beijing 199191, P.R. China \\ dongdayong@sjtu.edu.cn
}

\begin{abstract}
Data structure of the digital human model has been constructed for particularity demand of human factor evaluation of the load carriage system equipments. Anthropometry data of different percentage was obtained through regression calculation; Range of Motion (ROM) data of the different joints, data of upper limb reach zone and accessibility data of human body surface were also obtained by experiments. Reach zone envelop was constructed based on the experiment data. Different types of view cones have been constructed on the basis of related standard data to meet the use of human factor evaluation.
\end{abstract}

Keywords: Load carriage equipment, Simulation, Human factors, Digital Human model.

\section{Introduction}

With the development of computer visualization technology, the application of digital human model to carry out initial human factor evaluation of the design in the early digital stage of the system design has been gradually accepted by designers and developers. Presently, digital human model used in the design of both aircraft and automobile has become relatively universal. There is a Human Engineering module in CATIA which can build digital human model with different percentage height of different countries (e.g. America, France, Japan, etc.) for the evaluation on visibility and accessibility of digital model of aircraft system, and it can has a function of posture analysis.

Load carriage equipment is usually used by soldiers, police and outdoor travelers. However, it is generally attached to human body surface and varies with human motion, which is of great difference compared with driving environment in the 
aircraft or car where the pilot or the driver usually work in sitting posture. The equipment used in the cockpit for human-machine interaction does not move with human body, therefore, digital human model enjoys distinctiveness when used in load carriage system design evaluation. Research has been carried out aiming at this distinctiveness. First, human model structure is built with 66 segments, 65 joints and 131 freedom; secondly, different percentage anthropometry data is built by regression calculation on the basis of GJB (China's military standards) original data; thirdly, the range of joint motion and accessibility data are determined based on experimental data; lastly, visibility data is determined based on GJB.

\section{Data Structure}

H-Anim standard is an international standard on virtual human model. Its full name is Humanoid Animation Specification. Such standard is established for expression of virtual human in online virtual environment, taking into account of compatibility, adaptability and conciseness. Current version of H-Anim 2001 processes description by using VRML 97[1].

H-Anim standard utilizes Prototype Support in VRML97, defining five types of custom nodes to describe virtual human model — they are Humanoid, Joint, Segment, Site and Displacer, among which, the node of joint is used for composing bone structure of virtual human

H-Anim standard divides the whole human body into 1 human body gravity, 77 joints and 47 bone segments - these elements constitutes an integrated virtual human model. Since virtual human bone segments are connected by joints, the motion of human gravity, every bone segment and joint will affect other node status related to it. This definition accords with physiological characteristics of human motion. $\mathrm{H}$-Anim standard hierarchy is realized by nested joint node. The sacroiliac joint at the end of spinal column is regarded as the root of the whole skeleton structure which will be traversed from top to bottom. All the joints will be organized into treeshaped inheritance structure according to the order of various joints encountered. Each joint node is a parent node of those ranking behind, which forms skeleton of human model.

This research is based on the above mentioned international standards and simplifies neck segments according to actual needs. It divides human body into 66 segments, 65 joints and 131 freedom, which includes: body (pelvis and 17 spines, totaling 18 segments; 12 thoracic vertebra and 5 lumber vertebra in 17 spines), left shoulder, right shoulder, neck, head, left upper arm, right upper arm, left front arm, right front arm, left hand (palm and 15 pieces of finger bones, totaling 16 segment), right hand (palm and 15 finger bones, totaling 16 segments), left thigh, right thigh, left shin, right shin, left foot ( 2 segments) and right foot (2 segments). Topological structure is shown as follows in fig 1 . 


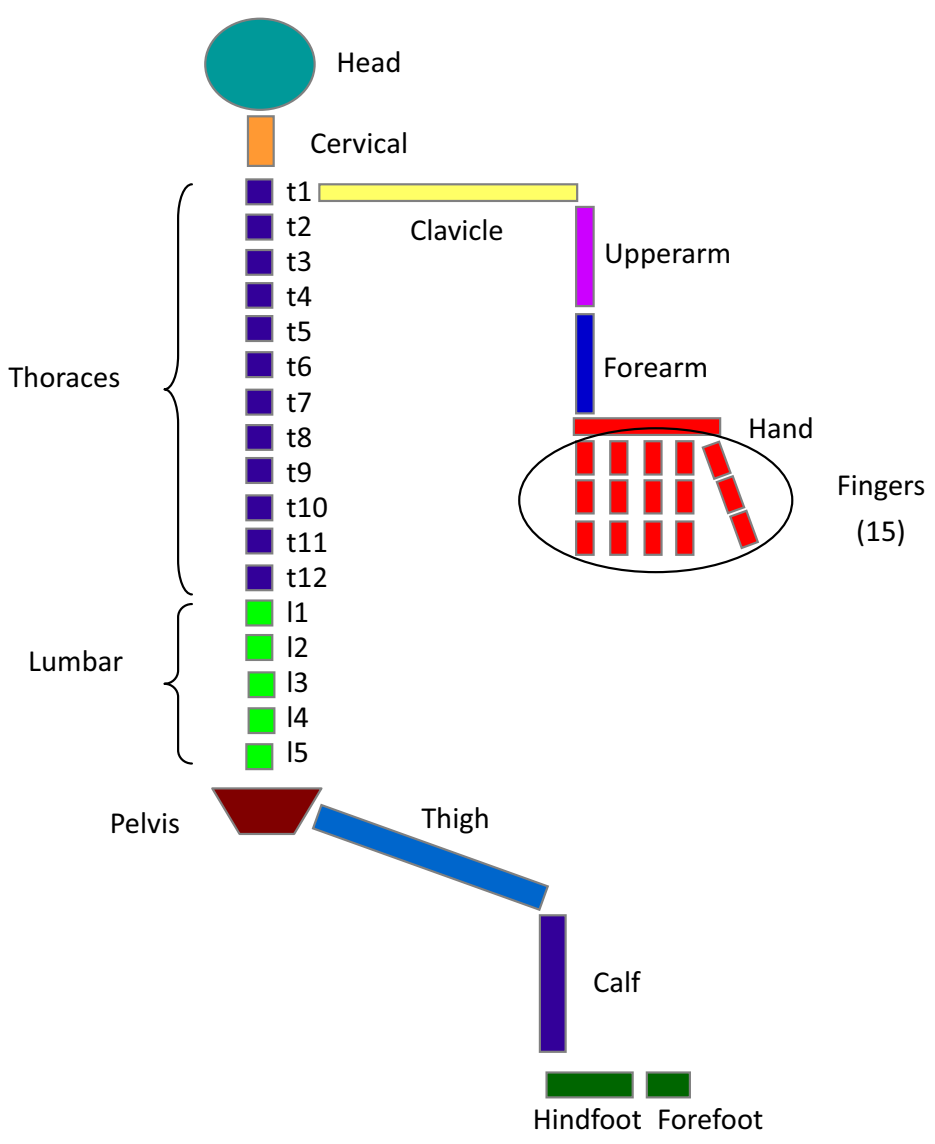

Fig. 1. Human Model Topological Structure

\section{Human Body Measurement Size}

When carrying out human body modeling, it is necessary to define the sizes of various segments according to human body measurement size. GJB4856-2003 provides basic size of part of percentages. In order to realize human body modeling of any percentages, Haiqiang Li from Beijing University of Aeronautics and Astronautics conducted research on relatedness between main human body measurement size and height and weight and figured out relevant regression parameters [2]. In this paper such kind of research findings were adopted to control human body modeling dimension so as to realize human model with different dimension as is shown in fig 2 . 


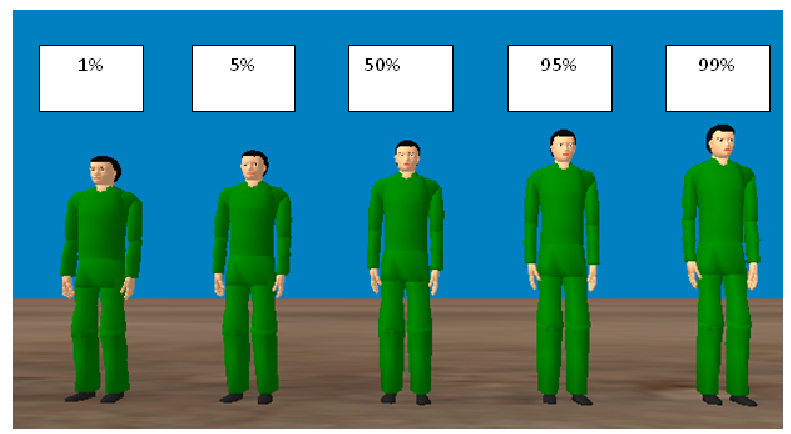

Fig. 2. Human Model of Different Percentages

\section{Range of Motion of the Joint}

The restraints which load carriage equipments exert on human joints mainly center on shoulders and body trunk. The study conducts experiment to carry on one's shoulder $20 \mathrm{~kg}$ backpack to simulate restrictions that load carriage system exerts on the joints. The shoulder straps of backpack are $80 \mathrm{~mm}$ wide and are used to measure the range of motion of the joints.

The data base for the range of motion of the joints is established to define ROM data sheet. The data structure is shown in table 1 below.

Table 1. Data Structure of Joint ROM

\begin{tabular}{ll}
\hline Field name & description \\
\hline Joint Name & Name of joint \\
$\mathrm{X} 1$ & Minimum value of $\mathrm{X}$ axis \\
$\mathrm{X} 2$ & Maximum value of $\mathrm{X}$ axis \\
$\mathrm{Y} 1$ & Minimum value of $\mathrm{Y}$ axis \\
$\mathrm{Y} 2$ & Maximum value of $\mathrm{Y}$ axis \\
$\mathrm{Z} 1$ & Minimum value of $\mathrm{Z}$ axis \\
$\mathrm{Z} 2$ & Maximum value of $\mathrm{Z}$ axis \\
\hline
\end{tabular}

In user interface, topological structure of human model joints are shown by means of human-computer interaction and joint angle can be adjusted manually, as is shown in fig 3 . 


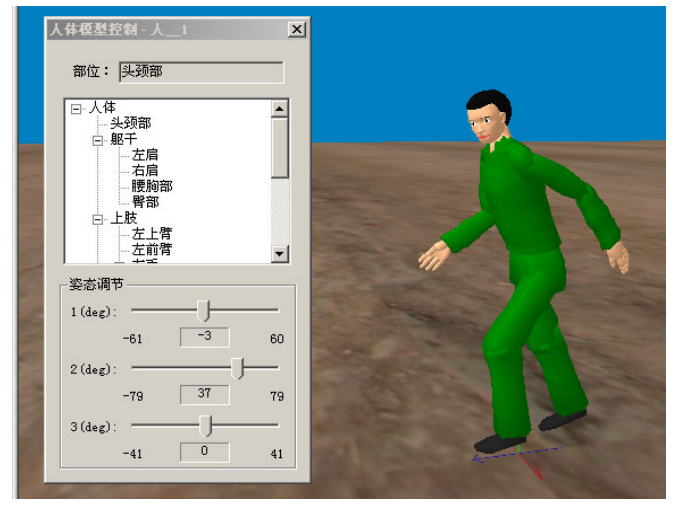

Fig. 3. Human Model Joint Adjustment

\section{Accessibility Data}

\subsection{Accessible Region Enveloping Surface}

First, let the subject stand at attention at original point; then, divide horizontal plane according to $15^{\circ}$ interval and draw $15^{\circ}$ interval line on the ground; and then, divide a plane every $10 \mathrm{~cm}$ in vertical direction and measure from middle fingertip that is naturally hanging down; after that, use a vertical scale ruler to guide the subject to perform touch actions and utilize VICON motion track system to let the subject perform actions in capture space; finally, record coordinate points of fingertips and use three-dimensional software to generate enveloping curve surface.

12 male subjects were selected to measure accessible region of their upper limbs. In order to measure maximum accessible region under free conditions as well as maximum accessible region and comfortable accessible region under the condition of restricted shoulder joints, the experiment carries out measurement on these three kinds of status:

1. Under free condition, that is, the subjects will not carry any equipment and shoulder joints will not be subjected to any restriction. The subjects will stretch their upper limbs as far as they can and perform touch actions under guidance;

2. Under the condition of restricted shoulder joints, that is, the subjects will carry load system with $80 \mathrm{~mm}$ wide shoulder straps and thus, the shoulder joints will somewhat be restricted by shoulder straps. The subject will stretch their upper limbs as far as they can and perform touch actions under guidance;

3. Stretch the upper limbs to a comfortable and reachable state after their shoulder joints are restricted, that is, the subjects will carry load system with $80 \mathrm{~mm}$ wide shoulder straps and thus, the shoulder joints will somewhat be restricted by shoulder straps. The subject will stretch their upper limbs comfortably as far as they can through their subjective feeling and perform touch actions under guidance; 
In order to avoid measurement error brought about by the fatigued subjects, the subjects will be arranged to take 15 minutes break after each collection of experimental data till all the data are collected. The generated enveloping surface is demonstrated in figure 4.
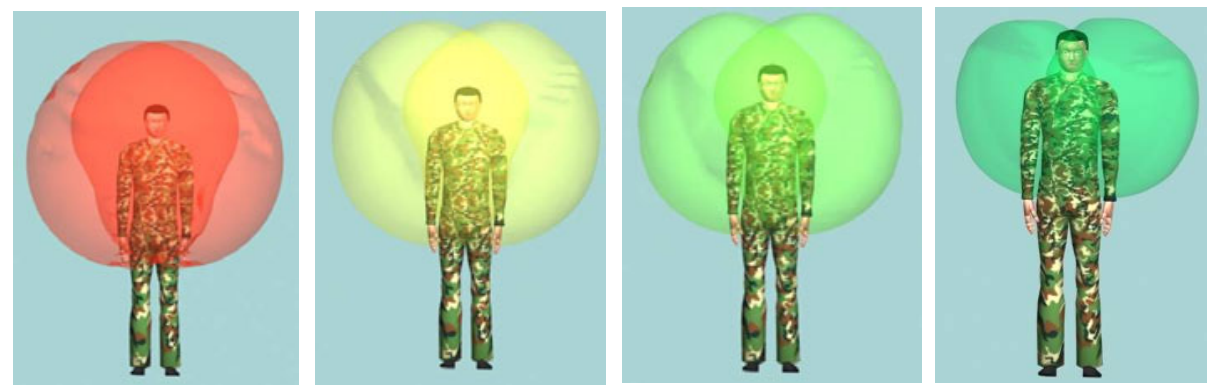

Fig. 4. Accessible Region Enveloping Surface

\subsection{Accessibility of Human Body}

Divide human body into 32 parts (see fig. 5). Select the subjects to present standing position, crouched position and creeping motion, to perform touch actions to different body parts and make subjective judgment to accessibility levels which can be divided into three: comfortably reachable, extremely accessible and inaccessible. Comfortably reachable means that the subjects can easily touch their body parts and will not generate uncomfortable feeling; extremely accessible means that the subjects can get in touch with their body parts, but will generate unconformable feeling; Inaccessible means that the subjects cannot reach any of their body parts. Experimental data is available in reference [3].

Experimental data can be mapped onto digital human model surface.
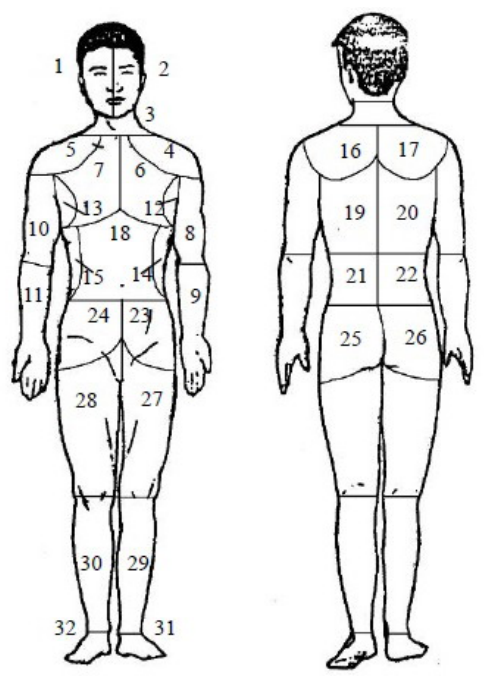

Fig. 5. Regional Division of Human Body 


\section{Visibility}

According to related regulations in GJB2873-97[4] and MIL-HDBK-759A[5], this paper constructs visual field in both plane and vertical direction to draw vision cone under three conditions: eye rotation, head rotation, head and eye rotation (Table 2). The different type of view cone of digital human model is shown in Fig 6.

Table 2. Visual Field

\begin{tabular}{lccc}
\hline View cone & Up & down & Left/right \\
\hline Preferred & 15 & 15 & 15 \\
Eye rotation only & 40 & 20 & 35 \\
Head rotation & 65 & 35 & 60 \\
Head and eye rotation & 90 & 75 & 95 \\
\hline
\end{tabular}
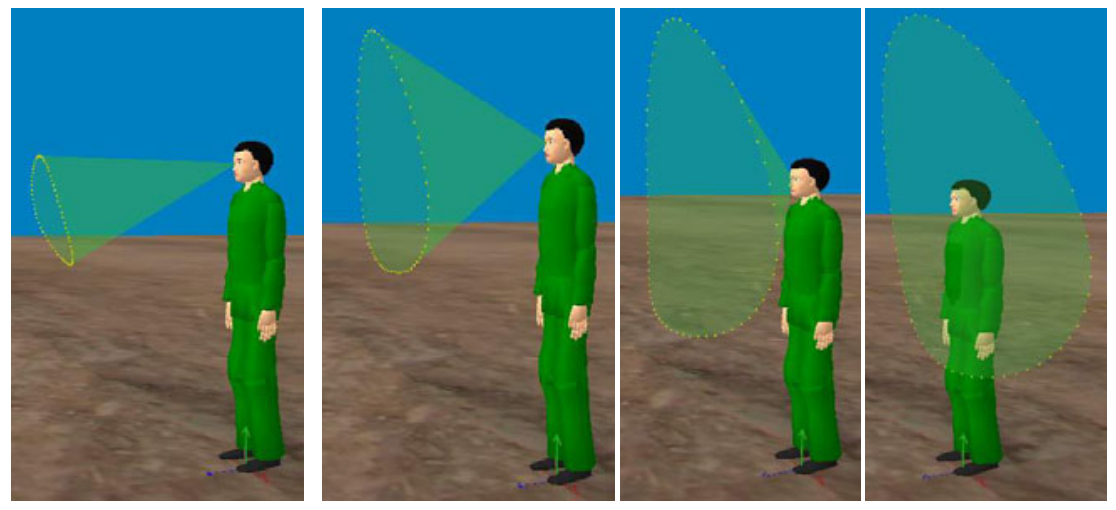

Fig. 6. View cone of digital human model

\section{Conclusion}

In this paper, the author introduced a digital human model used in human factor evaluation of load carriage equipments. Some experiments were carried on, and the data from the experiments were used in the human body modeling.

\section{Acknowledgement}

This work was supported by a grant from the National Basic Research Program of China (973 Program, No. 2010CB734103). 


\section{References}

1. International Organization for Standardization (ISO).The Humanoid Animation Specification. International Standard ISO/IEC FCD 19774:200X (2001)

2. Li, H.: The Correlation Analysis of Body Measurements for Human Model. BUAA (2007) (in Chinese)

3. Dong, D., Wang, L., Yuan, X.: Experimental Research on the Confirmation of Human Body Surface Reachable Grade. AHFE (2010)

4. Human engineering design criteria for military equipment and facilities. GJB2873-1997 (1997) (in Chinese)

5. MIL MIL-HDBK-759A. Human Factors Engineering Design for Army Material Military Handbook, U.S. (1982) 